\title{
The Horse's Morus can be more Dramatic than that of a Dog, about a Case on a Horse's Bite
}

\section{Ouazzani Nabil*, Grimi Talal, Aguenaw Omar, Fekhaoui Med Reda, Bassir Mohammed Reda, Boufetal Moncef, El Bardouni Ahmed, Berrada Mohammed Saleh}

Department of Orthopedic Surgery and Trauma, IBN SINA Hospital, Rabat, Morocco

*Corresponding Author: Ouazzani Nabil, Resident in Orthopedic and Traumatology Surgery, Department of Orthopedic Surgery and Trauma, IBN SINA Hospital, Rabat, Morocco.

Received: August 20, 2019; Published: August 28, 2019

\begin{abstract}
The management of animal bites is a medical and surgical emergency. It is an aggression always deep and infected. It imposes a stereotyped attitude that alone avoids the inevitable evolution towards abcdation and the dramatic consequences that may result.

In this work, we report a case of a 49-year-old man with no particular antecedents, who suffered from a horse's bite, presented to the emergency department with open trauma to his wrist, with deformity and abolition of the radial radial pulse, having benefited directly in the emergency's operated room of a trimming, cleaning of the wound, and a bone reconstruction at least of his wrist, by K-wire, and a complement by a medical treatment including a serum anti-tetanus human monoclonal antibodies with high neutralizing activity and anti-rabic, with a probabilistic antibiotherapy, adapted later according to the bacteriological exams [1].
\end{abstract}

Keywords: Horse's Bite; Surgical Treatment; Open Trauma of the Wrist

\section{Introduction}

Bites account for $1 \%$ of emergency center visits in the United States.

Mainly the $\operatorname{dog}(76 \%)$ and the cat (14\%), while that of the horse does not exceed $1 \%$.

The extremities are most often affected,. Although sometimes considered harmless, bites are often infected with the oral flora of the biter or, more rarely, with the cutaneous flora of the bite. Local complications may exceptionally require limb amputation. Systemic complications with significant morbidity can lead to death, as in case of infection with Capnocytophaga canimorsus in an asplenic patient [2].

\section{Case Presentation}

Here we report the case of a 49-year-old man, with no specific antecedents, who presented to the emergency department with a horse bite, resulting in open left wrist trauma. The general examination was normal with a stable haemodynamic and respiratory state, while the physical examination object a deformed left wrist with a cutaneous opening trauma classified 3 stage according to the classification of cauchoix and duparc, with an abolition of the radial pulse at the vascular examination, and a sensitif and motor deficenty interesting the radial's nerve territory (Figure 1).

the radiological examination shows a radiocarpal dislocation fracture of the wrist, with fracture of the external cunean of the radius (Figure 2), the biological assessment is normal, the patient was 
admitted to the emergency operating room, where he benefited from a surgical trimming with a washing and cleaning the wound, then a minimal osteosynthesis with a K-wire between the lunate bone and the scaphoid bone, a k-wire between the pyramidal bone and the lunate bone, and a last k-wire between the big bone, the lunate bone and the radius, and finally a k-wire for maintaining the reduction of the fracture of the external cunean (Figure 3).

A complement therapeutic by medical treatment has been associated with an anti-rabbic serum, an anti-tetanus vaccine, and an antibiotic treatment containing protected amoxicillin and gentamycin; the operative follow-ups were simple with a daily change bandage.

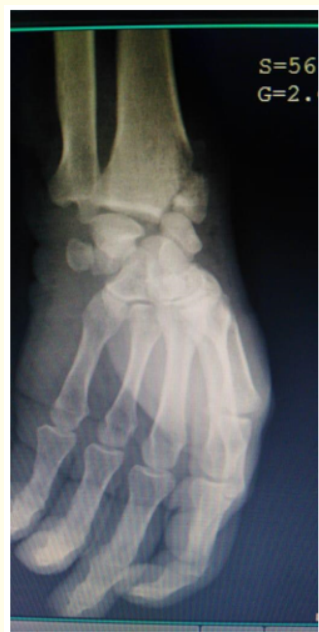

Figure 1

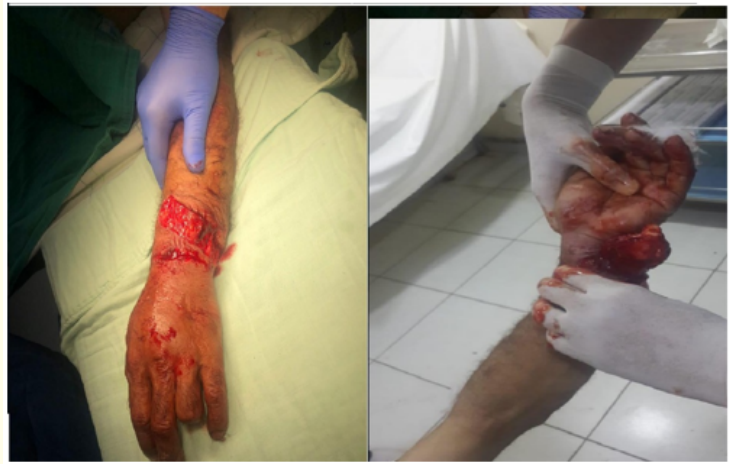

Figure 2

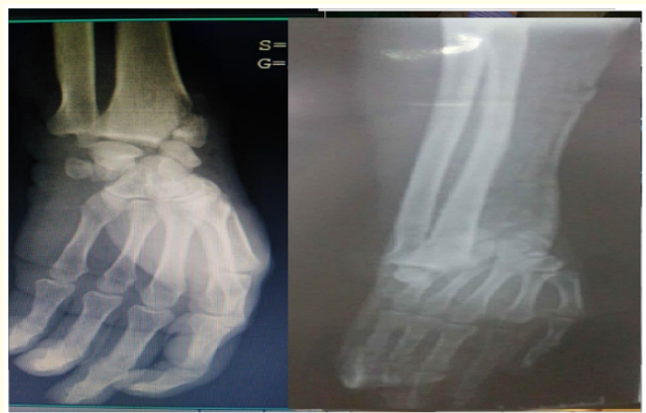

Figure 3

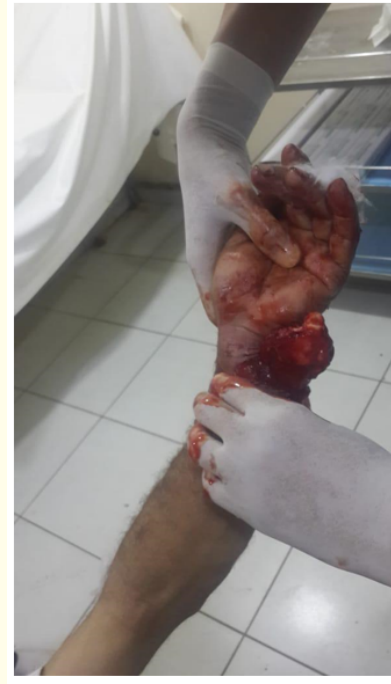

Figure 4

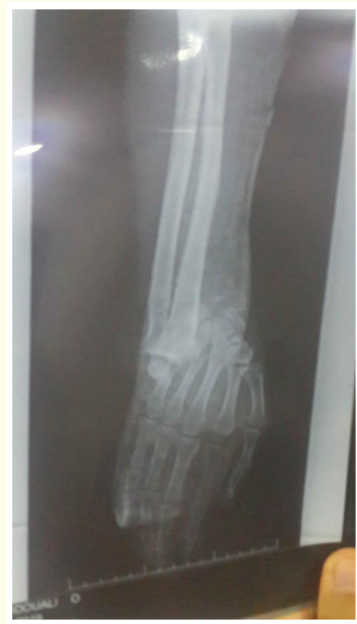

Figure 5 


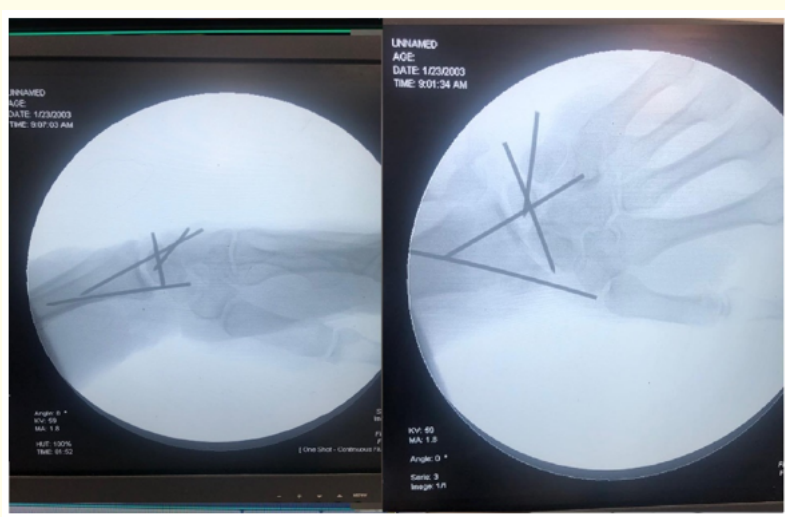

Figure 6

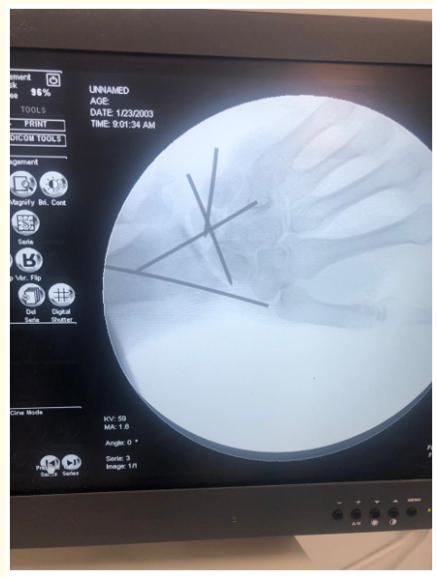

Figure 7

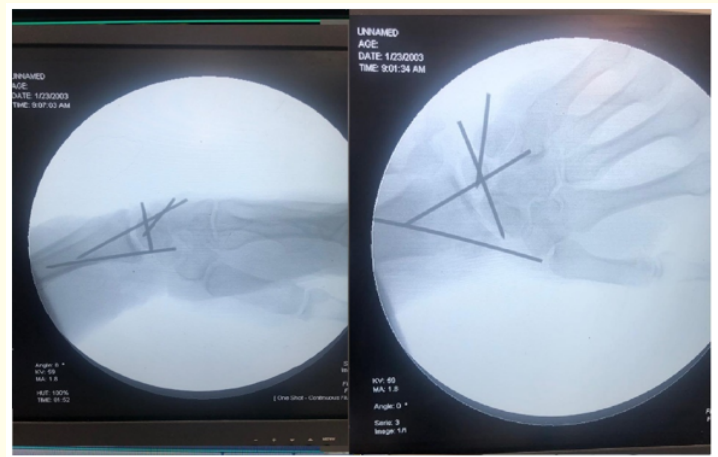

Figure 8

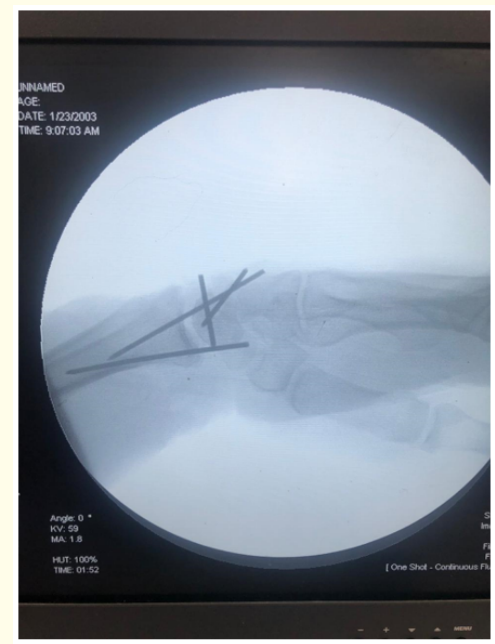

Figure 9

\section{Discussion}

Bacterial superinfections are the most common complications of animal bites. They occur on average 24 hours after a dog bite and 12 hours after a cat bite $[3,4]$ while there are no studies for the horse.

It is most often polymicrobial infections including aerobes and anaerobes [4].

These infections can be local (subcutaneous abscess, tendonitis, septic arthritis, osteomyelitis) or more rarely systemic (sepsis, endocarditis, meningitis, brain abscess) [5].

The following microorganisms are the most frequently involved: Pasteurella - staphylococcus and streptococcus - Moraxella - Corynebacterium - Neisseria - Bacteroides - tectum. Fusobacterium and Porphyromons - Capnocytophaga canimorsu [4]. In our case, there are two problems, an infectious problem that is the bite of the horse, and an orthopedic problem, which is the fracture-dislocation of the wrist, both of them constituted a surgical emergency, the speed of our care has prevented us from superinfection, and allowed an orthopedic reduction in the rules of art, the lesson to be learned from this case is that in front of these serious animal bites the time is in gold, the effectiveness of the surgical procedure is the rule, and when we have an alteration and disorganization of the skeletal system, do not hesitate to do an osteosynthesis, obviously 
covered by a probabilistic antibiotherapy, adapted later according to the examinations of bacteriological samples done at the operative room.

\section{Conclusion}

The bite of animals remains unpredictable in its evolution, according to the transmitted germs, according to its severity, its depth and then its location, the key to prevent complications is to reason according to an algorithm that addresses problem by problem, taking into account the risk of infection and aggression of the soft parts and the musculoskeletal system, not to mention the medical chapter which is the guarantor of a good evolution.

\section{Bibliography}

1. Conduite à tenir devant une morsure de chien (213b) Professeur Jacques LEBEAU Novembre 2003 (2005).

2. Noémie Boillat and Vincent Frochaux. "Morsures d'animaux et risque infectieux". Revue Médicale Suisse 4 (2008): 21492155 .

3. Mandell GL, Bennett JE, Dolin R. Bites. In: Mandell, Douglas, and Bennett's principles and practice of infectious diseases. New York : Churchill Livingstone 6th eds, (2006): 3552-3556.

4. Talan DA., et al. "Bacteriologic analysis of infected dog and cat bites. Emergency medicine animal bite infection study group". The New England Journal of Medicine 340 (1999): 85-92.

5. Weber DJ., et al. "Pasteurella multocida infections: Report of 34 cases and review of the literature". Medicine 63 (1984): 133-154.

Volume 2 Issue 9 September 2019

(C) All rights are reserved by Ouazzani Nabil., et al. 